\title{
Direct observation of acoustic phonon mediated relaxation between coupled exciton states in a single quantum dot molecule
}

\author{
T. Nakaoka, ${ }^{1,2}$ E. C. Clark, ${ }^{1}$ H. J. Krenner, ${ }^{1}$ M. Sabathil, ${ }^{1}$ M. Bichler, ${ }^{1}$ Y. Arakawa, ${ }^{2}$ G. Abstreiter, ${ }^{1}$ and J. J. Finley ${ }^{1}$ \\ ${ }^{1}$ Walter Schottky Institut and Physik Department, Technische Universität München, Am Coulombwall 3, 85748 Garching, Germany \\ ${ }^{2}$ Research Center for Advanced Science and Technology, University of Tokyo, 4-6-1 Komaba, Meguro-ku, Tokyo 153-8505, Japan
}

(Received 27 June 2006; published 25 September 2006)

\begin{abstract}
We probe acoustic phonon mediated relaxation between tunnel coupled exciton states in an individual quantum dot molecule in which the interdot quantum coupling and energy separation between exciton states is continuously tuned using static electric field. Time resolved and temperature dependent optical spectroscopy are used to probe interlevel relaxation around the point of maximum coupling. The radiative lifetimes of the coupled excitonic states can be tuned from $\sim 2 \mathrm{~ns}$ to $\sim 10 \mathrm{~ns}$ as the spatially direct and indirect character of the wave function is varied by detuning from resonance. Acoustic phonon mediated interlevel relaxation is shown to proceed over time scales comparable to the direct exciton radiative lifetime, indicative of a relaxation bottleneck for level spacings in the range $\Delta E \sim 3-6 \mathrm{meV}$.
\end{abstract}

DOI: 10.1103/PhysRevB.74.121305

PACS number(s): 78.67.Hc, 73.21.La, 71.35.Cc, 78.55.Cr

The charge and spin degrees of freedom of localized charges in quantum dot (QD) nanostructures currently attract significant interest due to their potential to form quantum bits for an inherently scalable quantum processor based on solidstate hardware. The implementation of such coherent devices using QDs can be traced to the strong three-dimensional confinement that gives rise to relatively long coherence times for charge $e^{1,2}$ and $\operatorname{spin}^{3,4}$ excitations when compared to the time required for quantum state manipulation. Very recently, advances in QD fabrication have allowed the realization of self-assembled double dot nanostructures in which the quantum coupling between electronic states, and the spatial localization of the exciton wave functions, can be continuously varied by varying the potential applied to a metal gate contact. $^{5-8}$ Such tunable QD molecules (QDMs) may allow ultrafast coherent manipulation using electro-optical techniques and may exhibit conditional coherent dynamics. ${ }^{9}$

A main obstacle to the realization of coherent QDM devices is decoherence caused by coupling to phonons. ${ }^{10-13}$ In pioneering work by Bockelmann ${ }^{14}$ phonon mediated relaxation between discrete electronic states in QDs was predicted to occur over time scales comparable to the radiative lifetime, an effect commonly termed the phonon bottleneck. However, experiments quickly revealed little or no evidence for bottleneck effects for level spacings of a few $10 \mathrm{~s}$ of $\mathrm{meV}$. These findings were explained by the participation of multiphonon processes, ${ }^{15}$ Coulomb scattering ${ }^{16}$ or polaron formation. ${ }^{17}$ In contrast, for level spacings of only a few meV, acoustic phonons have been shown to be the dominant source of decoherence for both single ${ }^{13,18}$ and vertically coupled $^{2,10-12,19}$ quantum dots. In this case the interlevel scattering rate can be strongly suppressed or enhanced, depending on the spatial extent of the carrier wave function. ${ }^{10}$ Therefore, experimental information on the phonon mediated coupling of the discrete states of a QDM is of fundamental importance for comparison with theory.

In this paper we probe acoustic phonon mediated interlevel relaxation between molecularlike coupled single exciton $(e+h)$ states in an individual self-assembled QDM. The interdot tunnel coupling is controlled by varying the axial electric field, enabling us to tune the coupled state energy splitting in the range $\Delta E \sim 3-6 \mathrm{meV}$ and control the mixing between spatially direct $(e+h$ localized in upper dot $)$ and indirect ( $e$ in lower dot, $h$ in upper dot) excitons. By combining continuous wave $(\mathrm{cw})$, time resolved and temperature dependent spectroscopy we demonstrate the ability to control the exciton spontaneous emission lifetime and probe acoustic phonon mediated relaxation as the level spacing and spatial character of the exciton wave function is varied.

The QDMs studied consisted of two layers of stacked InGaAs QDs with a nominal interdot separation of $d$ $=10 \mathrm{~nm}$. The molecules were embedded in an $n-i$ Schottky photodiode structure to control the static electric field $(F)$ along the growth axis and $\mathrm{Al}$ shadow masks were defined to optically isolate single QDMs. A schematic of the device structure is presented in the inset of Fig. 1(a). Further details of the InGaAs-GaAs QDM epitaxial layer sequence and sample fabrication can be found elsewhere. ${ }^{5,6}$ Time resolved studies were performed using a gated Si-APD and timecorrelated single photon counting techniques that provided a temporal resolution of $\lesssim 0.2 \mathrm{~ns}$. Carriers were photogenerated by a mode-locked Ti:sapphire laser tuned at $1.48 \mathrm{eV}$ to excite the QDM wetting layer that delivers $\sim 2$ ps duration pulses with an excitation power density of $4 \mathrm{~W} / \mathrm{cm}^{2}$.

A series of time integrated photoluminescence (PL) spectra recorded as a function of electric field $(F)$ are presented in the main panel of Fig. 1(a). A clear anticrossing between two transitions, labeled $X_{A}$ and $X_{B}$, can clearly be observed. These two peaks, which will be the focus of this paper, arise because of the coupling of spatially direct and indirect neutral excitons as discussed in our previous presentations. ${ }^{5,6}$ Other peaks observed, even at the lowest excitation powers investigated $\left(P_{\mathrm{ex}} \sim 0.1 \mathrm{~W} / \mathrm{cm}^{2}\right)$ also arise from the same QDM and arise from negatively charged excitons as discussed in Ref. 20 . At a low field $(F \lesssim 14 \mathrm{kV} / \mathrm{cm}) X_{B}$ is predominantly an unmixed state with spatially direct character. Thus, it shifts weakly with field and has a high electron-hole overlap and, consequently, a large oscillator strength. In contrast, $X_{A}$ is a state with predominantly indirect character over this low field range and thus shifts strongly with $F$ and has a 
(a)

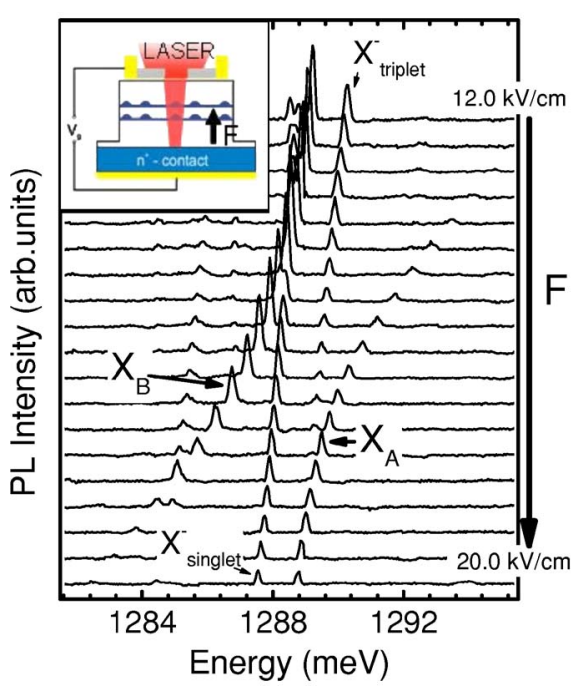

(b) $\quad F-F_{\text {crit }}(k V / c m)$

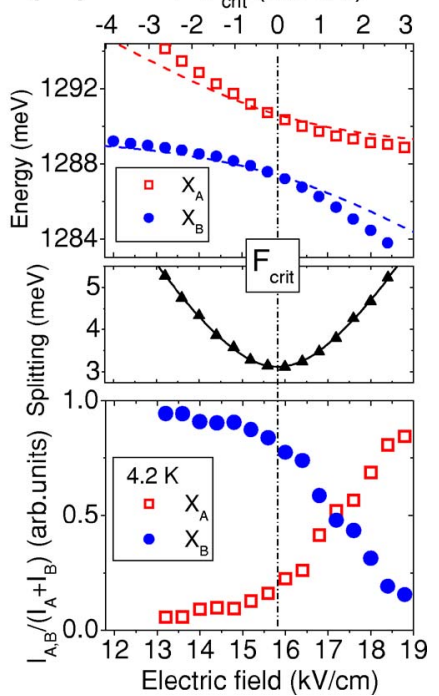

FIG. 1. (Color online) (a) PL spectra of a single QDM as a function of the electric field. (Inset) Schematic of the $n-i$ Schottky diodes studied. (b) Upper panel, experimental (symbols) and calculated (dashed lines) exciton energies for $X_{A}$ and $X_{B}$. Middle panel, energy splitting between $X_{A}$ and $X_{B}$ and fitting (see text). Lower panel, relative intensities $I_{A, B} /\left(I_{A}+I_{B}\right)$ and the resonant field (dashed line). weak oscillator strength. ${ }^{5}$ As the electric field increases, $X_{A}$ and $X_{B}$ shift closer to one another and become increasingly mixed until, for $F_{\text {crit }} \sim 15.8 \mathrm{kV} / \mathrm{cm}$, they anticross. At this point the electron component of the exciton wave function hybridizes over both dots to produce exciton states with bonding $\left(X_{B}\right)$ and antibonding $\left(X_{A}\right)$ character. The measured peak positions are compared with effective mass calculations of the coupled exciton states that include strain, quantum tunneling coupling, and Coulomb interaction for a model QDM with $d=11 \mathrm{~nm} .^{6}$ The results of this calculation are shown by the dashed lines on Fig. 1(b) (top panel), reproducing well both the field dependence of the transition energies of $X_{A}$ and $X_{B}$ and the coupling energy at $F_{\text {crit }}$ marked by a vertical line. Below, we use these effective mass calculations to obtain the $F$ dependence of the relative oscillator strengths of $X_{A}$ and $X_{B}$ and compare them to the results of our time resolved and cw spectroscopy. As expected for a coupled quantum system, the energy splitting between $X_{A}$ and $X_{B}$ is well represented by $\Delta E$ $=\sqrt{\left(2 \Delta E_{e-e}\right)^{2}+\left[\delta_{e-e}\left(F-F_{\text {crit }}\right)^{2}\right.}$ with $\delta_{e-e}\left(F-F_{\text {crit }}\right)$ being the detuning from the point of maximum coupling [Fig. 1(b), middle panel].

The lower panel of Fig. 1(b) shows the relative intensities $I_{A, B} /\left(I_{A}+I_{B}\right)$ of the upper and lower exciton branches as the resonance field is traversed. Clearly, the field at which the two exciton branches have equal relative intensities is larger than the critical electric field of $F_{\text {crit }}=15.8 \mathrm{kV} / \mathrm{cm}$. This observation contrasts strongly with the simple expectation that the radiative rates and populations are similar for both states at $F_{\text {crit }}{ }^{21}$ Moreover, it indicates the presence of a relaxation process from $X_{A}$ to $X_{B}$ that is active over time scales comparable to the radiative lifetime. We note that the observed intensity ratio of $I_{A} / I_{B} \sim 1: 5$ does not reflect a thermal population distribution since only the lower exciton branch would be significantly populated $\left[\exp \left(-2 \Delta E_{e-e} / k_{B} T\right)<10^{-4}\right]$.

In order to obtain further information about the nature of the relaxation process we performed time-resolved spectroscopy on the two exciton branches as a function of electric field. Typical PL decay traces recorded for $X_{A}$ and $X_{B}$ are presented in Fig. 2(a) for an electric field detuned $\left(F-F_{\text {crit }}\right.$ $=+2.6 \mathrm{kV} / \mathrm{cm})$ from the critical field. Under these conditions $X_{B}$ exhibits a much slower decay than $X_{A}$ since the spatial overlap of the electron and the hole is much weaker for the mixed exciton state due to its stronger fraction of indirect character.

Before entering into detailed analysis we present a summary of the decay lifetimes as a function of positive and negative detuning, either side of $F_{\text {crit }}$. From fitting the observed decay transients using single exponential decay functions we obtain the decay rates for $X_{A}$ and $X_{B}$ presented in Fig. 2(b). With increasing the electric field the decay rate measured for $X_{A}$ increases by a factor of $\sim 3 \times$ while the $X_{B}$ decay rate decreases by a similar factor. This distinct anticorrelation between the field dependent PL decay rates recorded for $X_{A}$ and $X_{B}$ demonstrates clearly that the electric field tunes the direct-indirect character of the two mixed excitonic states. States with strong indirect character have lower decay rates, increasing as the direct admixture becomes larger.

Surprisingly we find that $X_{A}$ and $X_{B}$ do not have the same
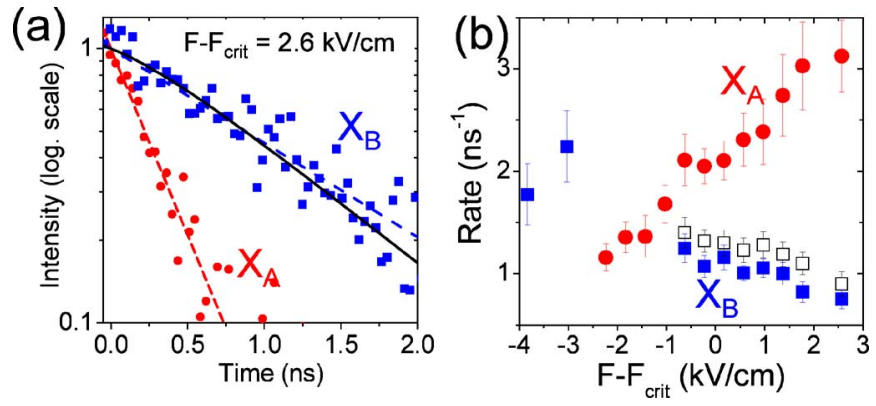

FIG. 2. (Color online) (a) Normalized PL decay curves for $X_{A}$ and $X_{B}$ measured at $F-F_{\text {crit }}=2.6 \mathrm{kV} / \mathrm{cm}$, plotted in a semilog scale. Dashed and the solid lines are fits with a monoexponential function and a biexponential function, respectively. (b) The PL decay rates for $X_{A}\left(D_{a}\right)$ (solid circles) and $X_{B}$ (solid squares) obtained by the monoexponential fittings. Total population decay rates for $X_{B}\left(D_{b}\right)$ (open squares) based on the rate equation model discussed in the text are also shown. 

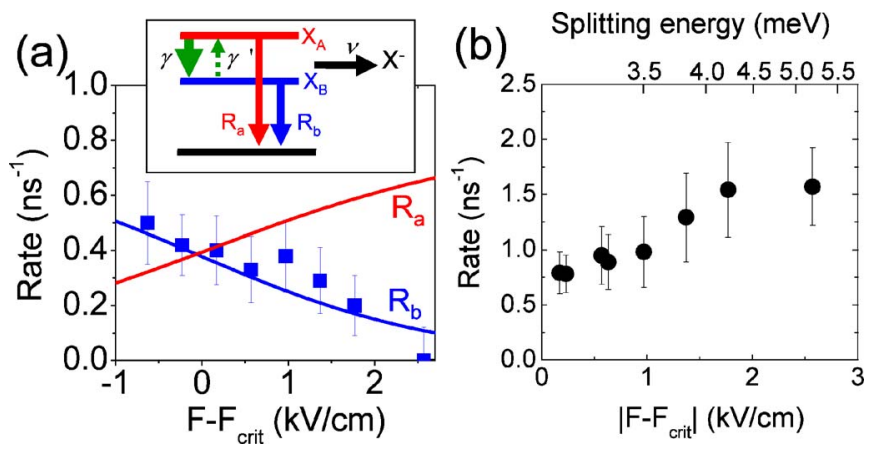

FIG. 3. (Color online) (a) Experimental (solid squares) and calculated (solid line) radiative recombination rates plotted as a function of $F$. (Inset) schematic processes for radiative recombinations of the exciton states $X_{A}$ and $X_{B}$, relaxation between them, and electron capture. (b) Phonon relaxation rate $\gamma=\gamma_{0}$ at $4.2 \mathrm{~K}$ from $X_{A}$ to $X_{B}$ obtained from the time-resolved PL and rate-equation model.

decay rate at $F_{\text {crit }}$ despite our simple expectation that the two states should carry similar oscillator strength. Instead, the observation of a different decay rate for $X_{A}$ and $X_{B}$ at $F_{\text {crit }}$ strongly indicates the existence of a nonradiative relaxation channel from the antibonding $\left(X_{A}\right)$ to the bonding state $\left(X_{B}\right)$ with a rate comparable to the pure radiative lifetime. In order to quantify the relaxation rate and radiative lifetime, we consider a simplified rate equation model illustrated by the three level system presented in the inset of Fig. 3(a). In this model, the two neutral exciton levels $X_{A}$ and $X_{B}$ decay radiatively with electric field dependent rates $R_{a}$ and $R_{b}$, respectively. The nonradiative relaxation between the upper and lower exciton branches are taken into account by emission $(\gamma)$ and absorption $\left(\gamma^{\prime}\right)$ rates due to phonon emission. In addition, a phenomenological electron capture process with rate $\nu$ is included due to the prominence of negatively charged excitons in the PL spectra presented in Fig. 1(a). The rate equations for the population of $X_{A}\left(N_{a}\right)$ and $X_{B}\left(N_{b}\right)$ subject to the processes discussed above can be set in the form

$$
\begin{aligned}
& \frac{d N_{a}}{d t}=-R_{a} N_{a}-\gamma N_{a}+\gamma^{\prime} N_{b}-\nu N_{a}+G, \\
& \frac{d N_{b}}{d t}=-R_{b} N_{b}-\gamma^{\prime} N_{b}+\gamma N_{a}-\nu N_{b}+G,
\end{aligned}
$$

where $G$ is the neutral exciton generation rate which is the same for $X_{A}$ and $X_{B}$. In order to simplify further our analysis, we note that $\gamma^{\prime} \ll \gamma=\gamma_{0}$ at $4.2 \mathrm{~K}$ since the phonon population is negligible $\left(<10^{-4}\right)$ and solve the rate equations to obtain the time dependence of $N_{a}$ and $N_{b}$, viz.

$$
\begin{gathered}
N_{a}=\exp \left(-D_{a} t\right), \\
N_{b}=(1+\alpha) \exp \left(-D_{b} t\right)-\alpha \exp \left(-D_{a} t\right) .
\end{gathered}
$$

Here $D_{a}=R_{a}+\nu+\gamma_{0}$ and $D_{b}=R_{b}+\nu$ are the measured decay rates for $X_{A}$ and $X_{B}$ and $\alpha=\gamma_{0} /\left(D_{a}-D_{b}\right)$. The decay rates extracted from a least squares fitting of monoexponential $\left(N_{a}\right)$ and biexponential $\left(N_{b}\right)$ transients are presented in Fig. 2(b) from which we estimate $\gamma_{0} \sim 0.6 \pm 0.5 \mathrm{~ns}^{-1}$. Here, the error is large as $\gamma_{0}$ is extracted from the relative amplitudes of the biexponential fit. Thus, we also estimated $\gamma_{0}$ by measuring the difference between $D_{a}$ and $D_{b}$ [Fig. 2(b)] at $F_{\text {crit }}$. This provides a direct measure of the interlevel relaxation rate since $R_{a}\left(F_{\text {crit }}\right) \approx R_{b}\left(F_{\text {crit }}\right)$ providing that the charged exciton formation rate is the same for both $X_{A}$ and $X_{B}$. This approach yields $\gamma_{0}\left(F_{\text {crit }}\right)=D_{a}\left(F_{\text {crit }}\right)-D_{b}\left(F_{\text {crit }}\right) \sim 0.7 \pm 0.3 \mathrm{~ns}^{-1}$, in good agreement with the value obtained by fitting the biexponential decay transient.

In order to investigate the $F$ dependence of $\gamma_{0}$ we need to estimate $\nu$, the electron capture rate. To do this we focus on the field range $-1 \mathrm{kV} / \mathrm{cm}<\left(F-F_{\text {crit }}\right)<3 \mathrm{kV} / \mathrm{cm}$, since the charged excitons are pronounced at lower field and $D_{b}$ can be directly measured in this region. We expect that the recombination rate of the indirect exciton $R_{b}$ is much smaller than $\nu$ in the high field range $(F>18 \mathrm{kV} / \mathrm{cm})$, since $X_{B}$ is much weaker than other lines arising from charged exciton recombination [Fig. 1(a)]. We then estimate $\nu=0.9 \mathrm{~ns}^{-1}$ from $D_{b}$, constant in this narrow field range since the field dependence of the charged exciton intensity is much weaker than the neutral exciton transitions. Using this value for $\nu$, we deduce the radiative recombination rate $R_{b}$. The results of this analysis are presented in Fig. 3(a) and compared with the calculated recombination rate obtained in the dipole approximation, i.e., $R=n_{r} e^{2} E_{\mathrm{exc}} E_{P}\left|\left\langle f_{e} \mid f_{h}\right\rangle\right|^{2} / 2 \pi \epsilon m_{0} c^{3} \hbar^{2}$, where $n_{r}=3.4$ is the refractive index, $E_{p}=25.7 \mathrm{eV}$ is the Kane matrix element, $E_{\text {exc }}$ is the emission energy, and $\left\langle f_{e} \mid f_{h}\right\rangle$ is the overlap integral of the envelope functions obtained from the effective mass calculations used to fit the transition energies in Fig. 1(b). Fairly good quantitative agreement is obtained between these calculations and the values of $R_{b}$ extracted from our rate equation analysis, strongly supporting the general validity of the analysis. The radiative lifetime can be tuned from $\sim 2$ to $\sim 10 \mathrm{~ns}$ as the fraction of direct character in the mixed exciton state is tuned, in good quantitative agreement with previous reports for vertically stacked QDs. ${ }^{22,23}$ From the extracted values of $R_{b}$ and $\nu$ we obtain the electric field dependence of $\gamma_{0}$ presented in Fig. 3(b). Typical values are of the order of $\gamma_{0} \sim 1 \mathrm{~ns}^{-1}$ for a level separation $\Delta E$ $=3-4 \mathrm{meV}$, consistent with recent theory for phonon mediated relaxation in QDMs with properties similar to those investigated here..$^{10,11}$

At elevated temperatures both relaxation and activation processes determine the steady state populations $N_{a}$ and $N_{b}$ and thus the resulting intensities of $X_{A}$ and $X_{B}\left(I_{A}\right.$ and $\left.I_{B}\right)$. Figure 4(a) shows the measured field dependence of the relative intensities at $4.2 \mathrm{~K}\left(\Delta E \gg k_{B} T, \gamma \gg \gamma^{\prime}\right)$ and $45 \mathrm{~K}(\Delta E$ $\left.\sim k_{B} T, \gamma \sim \gamma^{\prime}\right)$. At $45 \mathrm{~K}, I_{A} \sim I_{B}$ for $F \sim F_{\text {crit }}$ as expected since $R_{a} \sim R_{b}$ at $F_{\text {crit }}$ [Fig. 3(b)] and the activation process results in $N_{a} \sim N_{b}$. This process is reproduced by our rate equation model with single phonon scattering processes. Solving Eqs. (1) and (2) in steady state we obtain the PL intensity ratio for a $\mathrm{cw}$ excitation at zero temperature, i.e.,

$$
\frac{I_{A}}{I_{B}}=\frac{1+\left(\nu+2 \gamma^{\prime}\right) / R_{b}}{1+(\nu+2 \gamma) / R_{a}} .
$$

At finite temperature, one-phonon emission (absorption) rates are given by $\gamma(T)=\gamma_{0}\left[N_{\mathrm{ph}}(T)+1\right]\left[\gamma^{\prime}(T)=\gamma_{0} N_{\mathrm{ph}}(T)\right]$, 


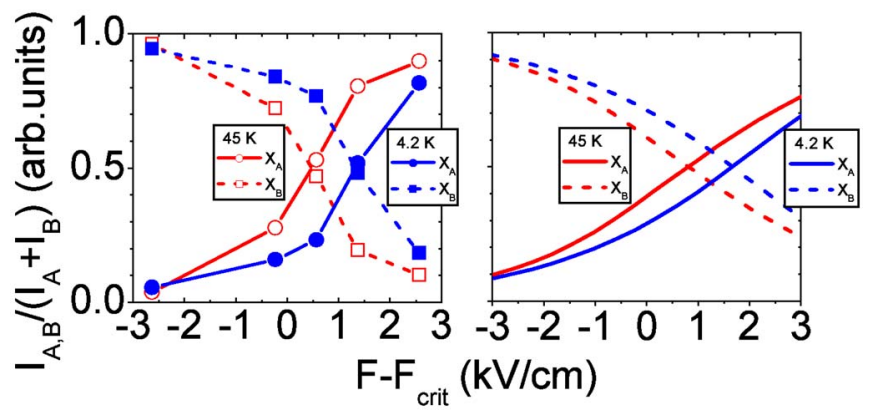

FIG. 4. (Color online) (a) Experimental and (b) calculated relative intensities of $X_{A}$ and $X_{B}$ at $4.2 \mathrm{~K}$ and $45 \mathrm{~K}$.

where $N_{\mathrm{ph}}(T)$ is the phonon occupation factor for the energy splitting between $X_{A}$ and $X_{B}$. The intensity ratios and their temperature dependence obtained by substituting the calculated $R_{a}, R_{b}$ and the estimated $\nu$ into the above equation are presented in Fig. 4(b), producing fairly good qualitative agreement with experiment. In particular, the shift of the equal intensity point towards $F \sim F_{\text {crit }}$ is reproduced. The broad agreement between the intensity ratios obtained from the cw PL measurement and calculation strongly supports the validity of our rate equation analysis and the assumptions made.

In summary, we have demonstrated the existence of interlevel phonon relaxation between the molecularlike exciton states in an individual self-assembled QDM. The relaxation rate is found to be comparable to the radiative recombination rates of the direct excitons demonstrating the existence of a phonon bottleneck in the meV range. Furthermore, we tune the radiative recombination rates of a single molecule as the fraction of the direct exciton character is varied. The observation and the calculation of the thermal evolutions of the exciton populations suggests a dominance of one-phonon processes for the interlevel relaxation in the QDM, with an intrinsic rate $\sim 0.5-1.5 \mathrm{~ns}^{-1}$.

This work was supported in part by the IT program (RR2002) from the MEXT (Japan) and by the DPG via SFB631 (Germany).
${ }^{1}$ A. Zrenner, E. Beham, S. Stufler, F. Findeis, M. Bichler, and G. Abstreiter, Nature (London) 418, 612 (2002).

${ }^{2}$ T. Hayashi, T. Fujisawa, H. D. Cheong, Y. H. Jeong, and Y. Hirayama, Phys. Rev. Lett. 91, 226804 (2003).

${ }^{3}$ M. Kroutvar, Y. Ducommun, D. Heiss, M. Bichler, D. Schuh, G. Abstreiter, and J. Finley, Nature (London) 432, 81 (2004).

${ }^{4}$ For a review, see, V. Cerletti, W. A. Coish, O. Gywat, and D. Loss, Nanotechnology 16, R27 (2005).

${ }^{5}$ H. J. Krenner, S. Stufler, M. Sabathil, E. C. Clark, P. Ester, M. Bichler, G. Abstreiter, J. J. Finley, and A. Zrenner, New J. Phys. 7, 184 (2005).

${ }^{6}$ H. J. Krenner, M. Sabathil, E. C. Clark, A. Kress, D. Schuh, M. Bichler, G. Abstreiter, and J. J. Finley, Phys. Rev. Lett. 94, 057402 (2005).

${ }^{7}$ G. Ortner, M. Bayer, Y. Lyanda-Geller, T. L. Reinecke, A. Kress, J. P. Reithmaier, and A. Forchel, Phys. Rev. Lett. 94, 157401 (2005).

${ }^{8}$ E. A. Stinaff, M. Scheibner, A. S. Bracker, I. V. Ponomarev, V. L. Korenev, M. E. Ware, M. F. Doty, T. L. Reinecke, and D. Gammon, Science 311, 636 (2006).

${ }^{9}$ J. M. Villas-Bôas, A. O. Govorov, and S. E. Ulloa, Phys. Rev. B 69, 125342 (2004).

${ }^{10}$ J. I. Climente, A. Bertoni, G. Goldoni, and E. Molinari, Phys. Rev. B 74, 035313 (2006).

${ }^{11}$ E. A. Muljarov, T. Takagahara, and R. Zimmermann, Phys. Rev. Lett. 95, 177405 (2005).

${ }^{12}$ P. Borri, W. Langbein, U. Woggon, M. Schwab, M. Bayer, S.
Fafard, Z. Wasilewski, and P. Hawrylak, Phys. Rev. Lett. 91, 267401 (2003).

${ }^{13}$ P. Borri, W. Langbein, S. Schneider, U. Woggon, R. L. Sellin, D. Ouyang, and D. Bimberg, Phys. Rev. Lett. 87, 157401 (2001).

${ }^{14}$ U. Bockelmann and G. Bastard, Phys. Rev. B 42, 8947 (1990).

${ }^{15}$ T. Inoshita and H. Sakaki, Phys. Rev. B 46, 7260 (1992).

${ }^{16}$ B. Ohnesorge, M. Albrecht, J. Oshinowo, A. Forchel, and Y. Arakawa, Phys. Rev. B 54, 11532 (1996).

${ }^{17}$ V. Preisler, T. Grange, R. Ferreira, L. A. de Vaulchier, Y. Guldner, F. J. Teran, M. Potemski, and A. Lemaître, Phys. Rev. B 73, 075320 (2006).

${ }^{18}$ T. Fujisawa, D. G. Austing, Y. Tokura, Y. Hirayama, and S. Tarucha, Nature (London) 419, 278 (2002).

${ }^{19}$ G. Ortner, R. Oulton, H. Kurtze, M. Schwab, D. R. Yakovlev, M. Bayer, S. Fafard, Z. Wasilewski, and P. Hawrylak, Phys. Rev. B 72, 165353 (2005).

${ }^{20}$ H. J. Krenner, E. C. Clark, T. Nakaoka, M. Bichler, C. Scheurer, G. Abstreiter, and J. J. Finley, Phys. Rev. Lett. 97, 076403 (2006).

${ }^{21}$ Our calculation gives the recombination-rate difference of $4 \%$ between $R_{a}$ and $R_{b}$ at $F_{\text {crit }}$.

${ }^{22}$ C. Bardot, M. Schwab, M. Bayer, S. Fafard, Z. Wasilewski, and P. Hawrylak, Phys. Rev. B 72, 035314 (2005).

${ }^{23}$ B. D. Gerardot, S. Strauf, M. J. A. de Dood, A. M. Bychkov, A. Badolato, K. Hennessy, E. L. Hu, D. Bouwmeester, and P. M. Petroff, Phys. Rev. Lett. 95, 137403 (2005). 